\title{
Normal and Oxidized Low Density Lipoproteins Accumulate Deep in Physiologically Thickened Intima of Human Coronary Arteries
}

\author{
Mitsumasa Fukuchi, Jun Watanabe, Koji Kumagai, Shigeo Baba, \\ Tsuyoshi Shinozaki, Masahito Miura, Yutaka Kagaya, and Kunio Shirato \\ Department of Cardiovascular Medicine, Tohoku University, Graduate School of Medicine, Sendai, Japan
}

\begin{abstract}
SUMMARY: Diffuse intimal thickening (DIT) that develops as a physiologic adaptation in the arterial wall has been implicated to have a predilection for atherosclerosis. We histologically investigated the lipid accumulation process in the human coronary DIT by focusing on the localization of normal and oxidized low-density lipoproteins (LDLs). Immunohistochemistry for apolipoprotein B 100 (a major apolipoprotein of LDL) and 8-iso-prostaglandin $F_{2 \alpha}$ (an oxidative product in LDL) showed substantial accumulation of oxidized relative to normal LDLs in the deep layers of DIT (52/139 segments). Subendothelial deposition of normal rather than oxidized LDLs, known as an early event of fatty streak formation, was less frequently found (13/139 segments). In contrast with fibrofatty lesions, lipid accumulation localized deep in DIT was characterized by fine lipid droplets scattered in the preserved tissue and by its association with neither macrophage accumulation nor apoptosis in the constituent cells. On the other hand, the deep intimal location of lipid accumulation clearly coincided with increased type I and type III collagen and elastic fibers but rarely with sulfated proteoglycans including decorin, which were all strongly expressed in advanced lesions. This lipid accumulation was found only in sites with DIT of more than $200 \mu \mathrm{m}$, occasionally extending to the inner media and involving neovessel formation around it. The presence of deep intimal lipid accumulation was associated with reduced endothelium-dependent relaxation to substance $\mathrm{P}$ in isolated coronary rings. These results suggest that normal and oxidized LDLs accumulate preferably in the nutritional border zone of established DIT involving local extracellular matrix alterations but independently of inflammatory or apoptotic processes. This may contribute to the functional and morphologic abnormalities seen in human coronary atherogenesis that progresses slowly with age. (Lab Invest 2002, 82:1437-1447).
\end{abstract}

7 he atherogenic mechanism initiated by endothelial injury or dysfunction leading to a series of inflammatory and proliferative responses in the intima is known as the response to injury theory (Ross, 1999). This hypothesis would be true particularly in experimentally induced atherosclerosis such as ballooninjury models (Schwartz et al, 1995). In human coronary arteries, however, early atherosclerotic lesions including fatty streaks begin in adolescence and slowly but steadily progress even until 70 years of age (Eggen and Solberg, 1968; PDAY Research Group, 1993; Stary, 1989). It is well known that primary atherosclerotic lesions tend to occur in sites with preexisting intimal thickening that consists of smoothmuscle hyperplasia (Montenegro and Eggen, 1968; Neufeld et al, 1962; Schwartz et al, 1995; Stary et al, 1992). This intimal hyperplasia is characteristic of human coronary arteries, forming diffuse intimal thickening (DIT) in most juveniles and is regarded as an adaptive response to mechanical stresses secondary to variations in coronary flow and wall tension with

\section{DOI: 10.1097/01.LAB.0000032546.01658.5D}

Received July 23, 2002.

Address reprint requests to: Dr. Kunio Shirato, Department of Cardiovascular Medicine, Tohoku University, Graduate School of Medicine, 1-1 Seiryo-machi, Aoba-ku, Sendai, 980-8574, Japan.E-mail: shirato@int1.med.tohoku.ac.jp every myocardial contraction (Ikari et al, 1999; Neufeld et al, 1962; Sims, 1989; Velican and Velican, 1979).

Although the atherogenic process initiated in DIT remains unclear, Ross's theory (Ross, 1999) has been challenged by some findings specific in human coronary arteries. First, no apparent alterations have been shown in the endothelium overlying human coronary DIT (Fukuchi and Giaid, 1999; Williams and Tabas, 1995). In addition, it has been reported that the rates of smooth muscle cell proliferation are not increased in either DIT or atherosclerotic plaques compared with that in the media (Gordon et al, 1990). It has been also reported that endothelium-dependent relaxation is impaired in proportion to the extent of DIT in isolated human coronary arteries (Keitoku et al, 1990). These findings lead us to speculate that some atherosclerotic changes proceed slowly beneath the DIT without active inflammatory and proliferative responses.

Previously, in the grossly normal human aortic intima, lipoprotein particles have been shown to accumulate along fibrous tissue elements, termed perifibrous lipid (Guyton et al, 1985; Smith et al, 1967). Lipid-retentive properties in the normal but thickened intima have been suggested to involve extracellular matrix molecules or local metabolic abnormalities (Adams and Bayliss, 1969; Guyton et al, 1985; Schwartz et al, 1995; Smith et al, 1967; Spring and Hoff, 1989; Stary et al, 1992; Williams and Tabas, 1995; Yamauchi and Hoff, 1984). Hitherto, it 
has not been explored how lipid accumulation occurs with the progression of DIT in human coronary arteries. In the present study, we histologically characterized the accumulation of normal and oxidized low-density lipoproteins (LDLs) in the human coronary DIT. Normal LDL was identified by using immunohistochemistry for apolipoprotein B 100 (apo B 100), a major apolipoprotein of $\mathrm{LDL}$. Oxidized lipids were detected by the presence of 8-iso-prostaglandin $\mathrm{F}_{2 \alpha}$ (8-iso-PGF ${ }_{2 \alpha}$ ) that can be synthesized in situ on phospholipids via a cyclooxygenaseindependent, free radical catalyzing pathway (Lynch et al, 1994; Morrow et al, 1992). Furthermore, we investigated the structural and cellular alterations associated with the lipid accumulation, especially focusing on the possible role of inflammatory responses, apoptosis, and extracellular matrix alterations. Finally, we investigated the pathologic aspects of DIT involved with lipid accumulation by correlating it with the extent of endotheliumdependent relaxation in isolated human coronary arteries.

\section{Results}

DIT was found in 30 of 40 coronary arteries (139/275 segments) examined. Among 30 arteries containing DIT, 16 arteries were also involved with fibrofatty or more advanced lesions in other sites of the same arterial course examined. The incidence of DIT among all coronary segments was irrespective of age (32-79 years old), and all DIT segments were pooled together to analyze the data.

Focal to extensive immunostaining for apo B 100 or 8-iso-PGF $2 \alpha$ was found in nearly half the DIT segments examined (71/139 segments). This lipid accumulation was prevalent even in patients with no coronary risk factors (47/97 segments). In most cases, apo B 100 or 8-iso-PGF ${ }_{2 \alpha}$ was localized either in the subendothelial space or in the deep intimal layers adjacent to the internal elastic lamina (Fig. 1). In the remaining cases, the stainings were distributed widely throughout the intima. In no types of lipid accumulations were there morphologic evidence of necrotic core formations. Both apo B 100 and 8-iso-PGF $2 \alpha$ stainings were significantly more prevalent in the deep intimal layers than in the subendothelial space (Fig. 2A). However, the subendothelial-deep intimal prevalence of apo B 100 was significantly higher than that of 8-iso-PGF ${ }_{2 \alpha}(n=18 / 41$ versus $7 / 48 ; p<0.05)$. Pretreatment of the sections with $20 \mu \mathrm{M} \mathrm{CuCl} 2$ for 6 hours resulted in the loss of apo B 100 staining and strong, extensive 8-iso-PGF ${ }_{2 \alpha}$ staining in most lipidrich areas (Fig. 2B). This finding supports that immunoreactive apo B 100 and 8-iso-PGF ${ }_{2 \alpha}$ would be reciprocally distributed depending on the local oxidized state.

In any DIT segments with apparent staining for apo B 100 or 8 -iso-PGF $2 \alpha$, only a few or no macrophages positive to CD68 were present throughout the intima (Fig. 3, A, C, and E). Smooth muscle cellularity was preserved in the lipid-rich areas, sometimes forming a honeycomb-like structure (Fig. 3G). In fibrofatty lesions, dense staining for apo $\mathrm{B} 100$ or 8-iso-PGF ${ }_{2 \alpha}$ was found
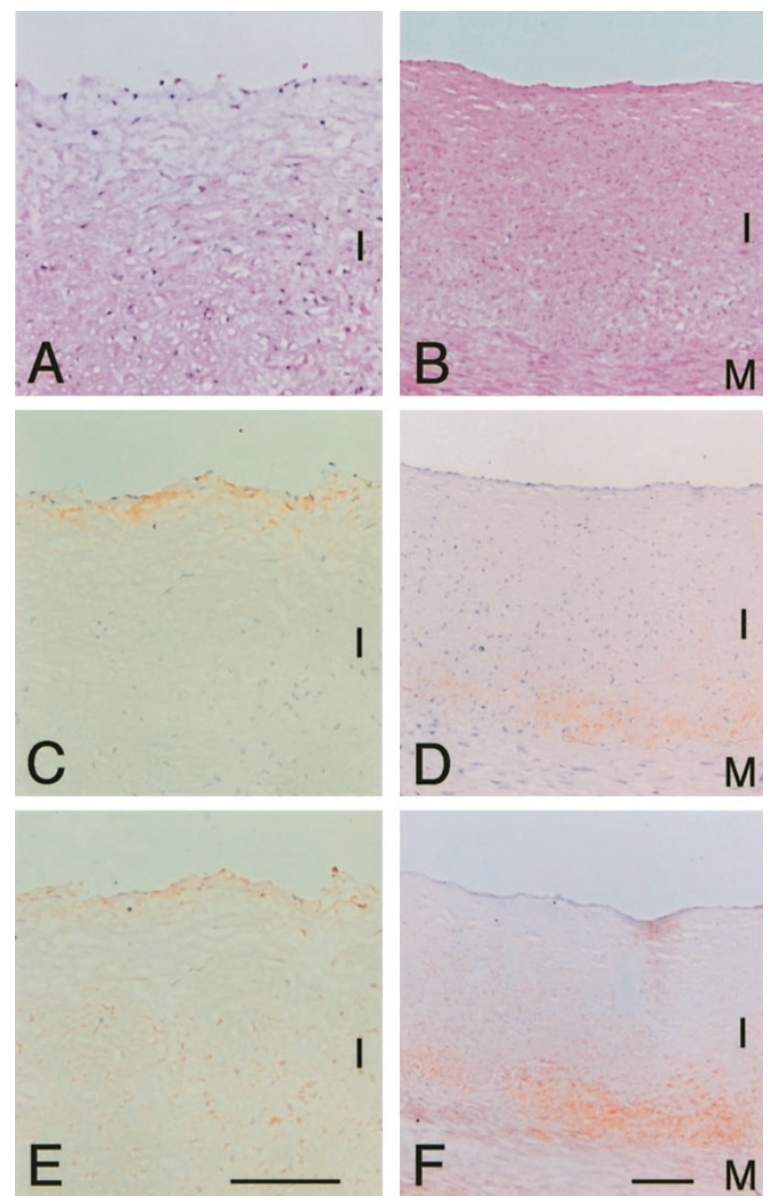

Figure 1.

Two typical immunohistochemic localizations of apolipoprotein B 100 (apo B $100)$ and 8-iso-prostaglandin $F_{2 \alpha}$ (8-iso- $\left.\mathrm{PGF}_{2 \alpha}\right)$ in diffuse intimal thickening (DIT) of human coronary arteries. A and B show hematoxylin and eosin staining. $C$ and $D$ show immunohistochemistry for apo B 100. E and $F$, immunohistochemistry for 8-iso- $\mathrm{PGF}_{2 \alpha}$. Bar $=100 \mu \mathrm{m} ; I=$ intima; $M=$ media.

in the hypocellular, lipid-rich core rather than in the region with macrophage accumulation (Fig. 3, B, D, F, and $\mathrm{H}$ ). Immunoreactive 8-iso-PGF ${ }_{2 \alpha}$ compared with apo B 100 was in general distributed deeper in the lipid-rich core (Fig. 3, B versus D). Oil red O staining showed fine lipid droplets scattered in the DIT with a distribution similar to that of immunoreactive apo B 100 or 8-iso-PGF ${ }_{2 \alpha}$ (Fig. 3I). In fibrofatty lesions, macrophages were filled with large lipid droplets (Fig. $3 \mathrm{~J}$ ) while fine lipid droplets were present in the extracellular space as seen in the DIT. The terminal deoxynucleotidyltransferase-mediated dUTP-fluorescein nick-end labeling (TUNEL) method showed no apoptotic cells in the deep intimal layers involved with lipid accumulation in any of the 25 DIT sections examined (Fig. 3K). Apoptotic cells (up to 10 cells per section) were found in the fibrofatty or advanced lesions (Fig. 3L,13/23 sections examined).

Next, we investigated the possible role of major LDL-retentive molecules in the lipid localization in DIT. Focal weak or no staining for type I and type III collagen and diffuse network of elastic fibers were generally found in DIT. Alcian blue staining was con- 

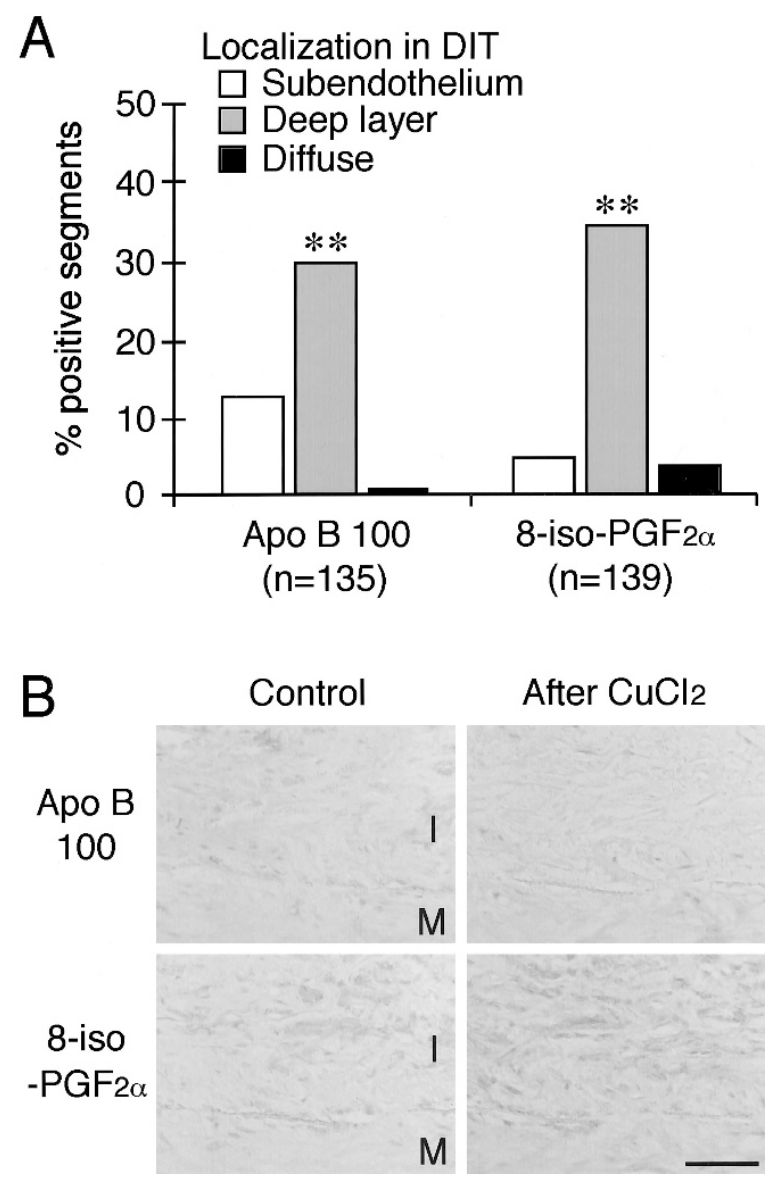

Figure 2.

Localization and prevalence of immunoreactive apo B 100 and 8-iso-PGF ${ }_{2 \alpha}$ in DIT. A, Summary of the prevalence of immunoreactive apo B 100 and 8 -iso-PGF ${ }_{2 \alpha}$ in terms of their localizations in DIT $\left({ }^{\star *} p<0.01\right.$ versus subendothelial site by chi-square analysis). B, Immunostaining for apo B 100 and 8-iso-PGF ${ }_{2 \alpha}$ in serial sections before (control) and after treatment with 20 $\mu \mathrm{M} \mathrm{CuCl} 2$ for 6 hours (after $\mathrm{CuCl}_{2}$ ). Bar $=50 \mu \mathrm{m} ; I=$ intima; $M=$ media.

sistently seen in the inner layers of DIT. However, apparent staining for decorin was seen in the media and adventitia but not in normal DIT. None of these stainings were associated with the subendothelial lipid deposition in DIT (Fig. 4A). On the other hand, lipid accumulation located deep in DIT clearly coincided with increased type I and type III collagen and elastic fibers but with reduced or no Alcian blue staining and no decorin staining in most cases examined (Fig. 4B). In advanced lesions, lipid accumulation around macrophages was associated with dense type I and type III collagen and decorin stainings but with variable density of elastic fibers (Fig. 4C). Interestingly, advanced lesions often involved a distinct lipid-rich layer located deeply in the plaques, which showed characteristics analogous to those seen in DIT (Fig. 4, B versus $\mathrm{C}$ ). Figure 4D summarizes the results of immunostaining for collagen type III, $\alpha$-elastin, and decorin associated with the location of lipid accumulation. These extracellular matrix molecules were differentially expressed between DIT and advanced lesions and even between the subendothelial and deep layers of DIT.
Because the deep intimal lipid accumulation was predominantly found around the thickest sites of DIT, we investigated the extent of intimal thickening critical for the occurrence of lipid accumulation. Figure 5 shows the distribution of intimal thickness at the thickest and thinnest sites in each DIT section in terms of the presence or absence of lipid accumulation. At both sites, intimal thickness was significantly greater in cases with, than in those without, lipid accumulation ( $p<0.01$ by unpaired Student's $t$ test). Although there was a wide scatter and marked overlap in the distribution, it was noteworthy that the apparent lipid accumulation was found only in sites with DIT greater than $200 \mu \mathrm{m}$. In cases with marked DIT, lipid accumulation in the deep intimal layers focally extended to the inner media, which was often characterized by reciprocal staining from apo $B$ 100-dominant to 8-isoPGF $_{2 \alpha}$-dominant toward the intima (Fig. 6, A, B, and D). In addition, neovessel formation was occasionally found around the lipid accumulation (Fig. 6C).

Figure 7A shows typical responses to the potent endothelium-dependent vasodilator, substance $P$, in human coronary rings with and without lipid accumulation in the DIT. A plot of the relation between the extent of DIT and the maximum relaxation to substance $P(10 \mathrm{~nm})$ was significantly different between cases with and those without lipid accumulation $(p<0.05$ by analysis of covariance; Fig. 7A). Immunostaining for endothelial nitric oxide synthase (eNOS) was preserved in the endothelium of most DIT sections (Fig. 7B). Only when DIT was greater than $400 \mu \mathrm{m}$ and further involved with lipid accumulation was eNOS expression slightly, but significantly, reduced ( $p<0.01$ vs comparable DIT sections by Mann-Whitney $U$ test). The endothelial expression of eNOS was progressively reduced in more advanced lesions (mean grade 1.84 in fibrofatty lesion, 1.81 in fibrous plaque, and 1.0 in advanced plaque; $p<0.01$ by Kruskal-Wallis rank test).

\section{Discussion}

The present study demonstrated substantial lipid accumulation in the deep layers of human coronary DIT, which was accompanied by neither macrophage accumulation nor apoptosis in the constituent cells. This lipid accumulation was characterized by the preferential 8-iso-PGF ${ }_{2 \alpha}$ relative to apo B 100 staining and by fine lipid droplets scattered in the preserved intimal tissue. The location of lipid accumulation clearly coincided with increased type I and type III collagen and elastic fibers but rarely with sulfated proteoglycans including decorin. The deep intimal lipid accumulation was found only in sites with DIT greater than $200 \mu \mathrm{m}$, occasionally involving the inner media and neovessel formation around it. The endothelium-dependent relaxation to substance $P$ was significantly reduced in the coronary arteries involved with deep intimal lipid accumulation. These observations characterize further the perifibrous lipid (Guyton et al, 1985; Smith et al, 1967), which had not been well recognized in the process of human atherosclerotic lesion development (Gown et al, 1986; Stary, 1989). 

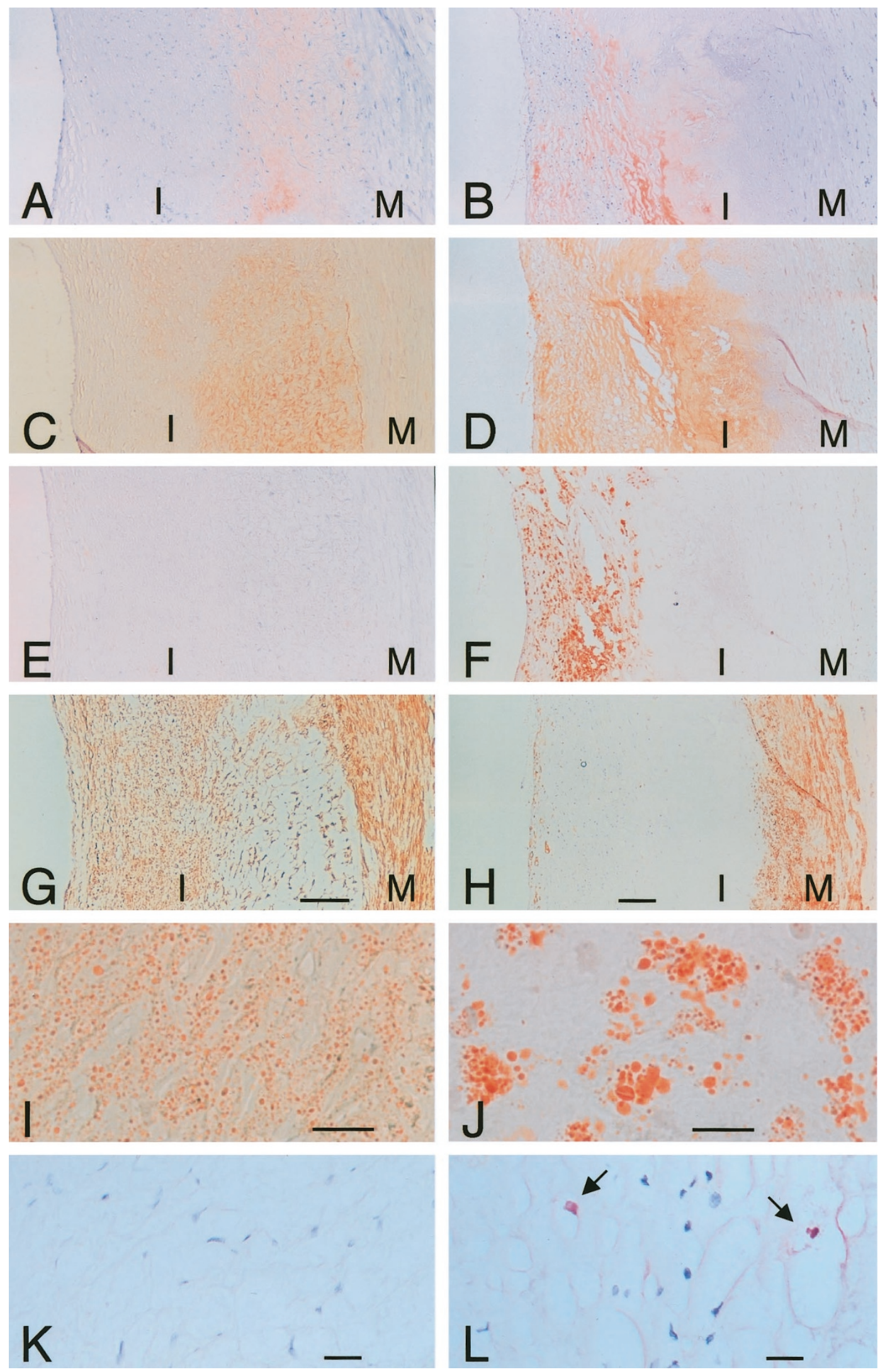

Figure 3.

Different histologic features between the lipid accumulation located deep in DIT (left panels) and the lipid-rich core in fibrofatty lesions (right panels). A and B show immunohistochemistry for apo B 100, C and D for 8-iso-PGF ${ }_{2 \alpha}$, E and F for CD68, and $\mathrm{G}$ and $\mathrm{H}$ for muscle actin. Bar $=100 \mu \mathrm{m} ; I=$ intima; $M=$ media. I and $J$ show oil red 0 staining, and $\mathrm{K}$ and $\mathrm{L}$ show the terminal deoxynucleotidyltransferase-mediated dUTP-fluorescein nick-end labeling (TUNEL) method in the lipid-rich areas of DIT and fibrofatty lesions, respectively. Arrows indicate the TUNEL-positive cells. Bar $=20 \mu \mathrm{m}$. 

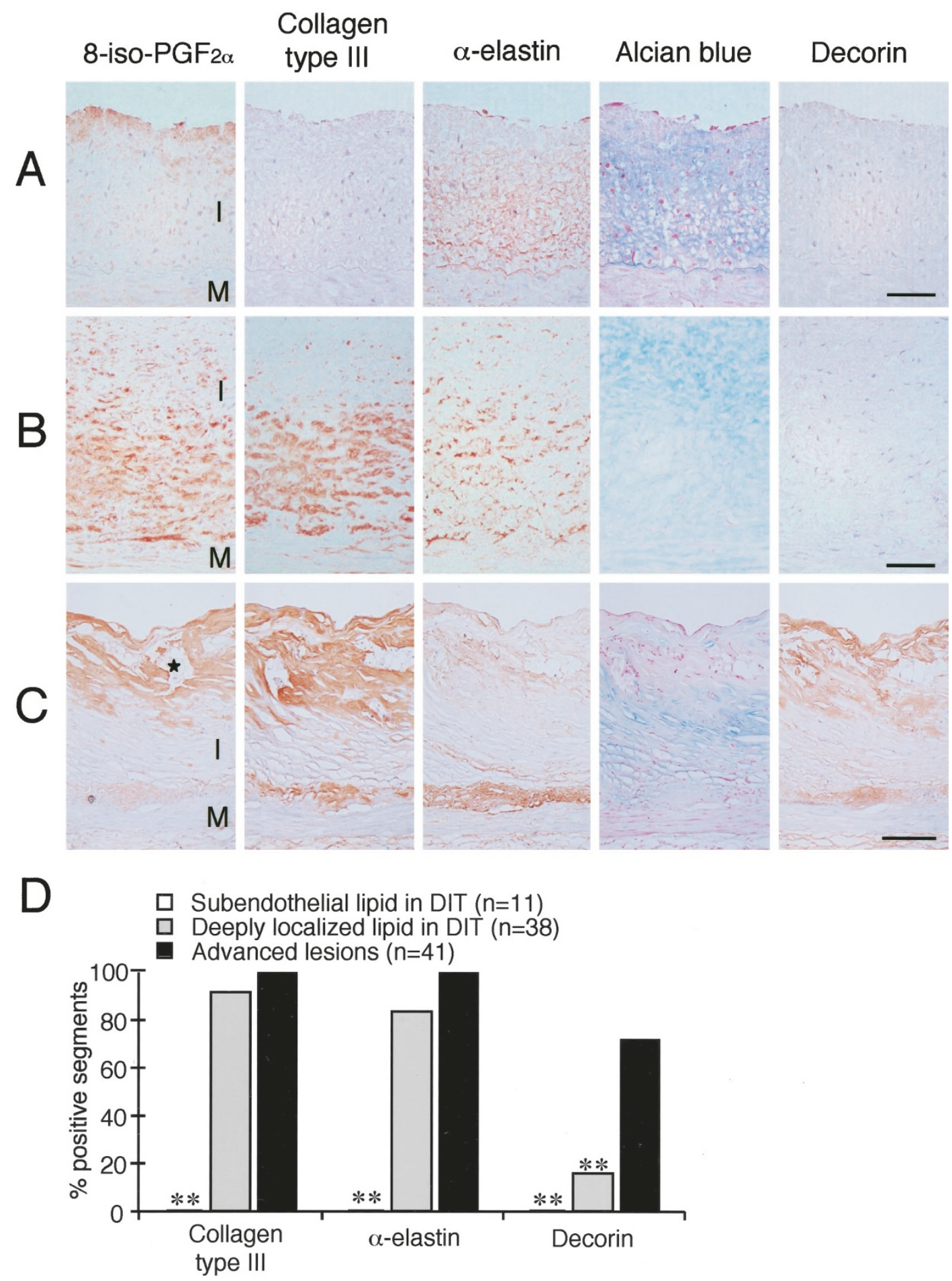

\section{Figure 4.}

Differential expression of extracellular matrix molecules associated with lipid accumulation in DIT and advanced lesions. A and B show serial sections of DIT in which lipid accumulation was localized in the subendothelial and deep layers, respectively. Bar $=50 \mu \mathrm{m}$. C shows serial sections of advanced plaque in which macrophages accumulated $\left({ }^{*}\right)$. Bar $=200 \mu \mathrm{m}$. Immunohistochemistry was performed against each epitope shown at the top of panels except for Alcian blue. Only the sections stained with Alcian blue were counterstained with nuclear fast red. I = intima; $M=$ media. D summarizes the positive staining for type III collagen, $\alpha$-elastin, and decorin associated with the location of lipid accumulation. ${ }^{* *} p<0.01$ versus advanced lesions by chi-square analysis.

\section{Lipid Accumulation in DIT}

Lipid deposition in the subendothelium is an early event of fatty streak formation in human and animal arteries (Napoli et al, 1997; Yamauchi and Hoff, 1984). In the present study, this type of lipid deposition was seen only in limited human coronary sections with DIT. In most cases, lipid accumulation was localized in the 


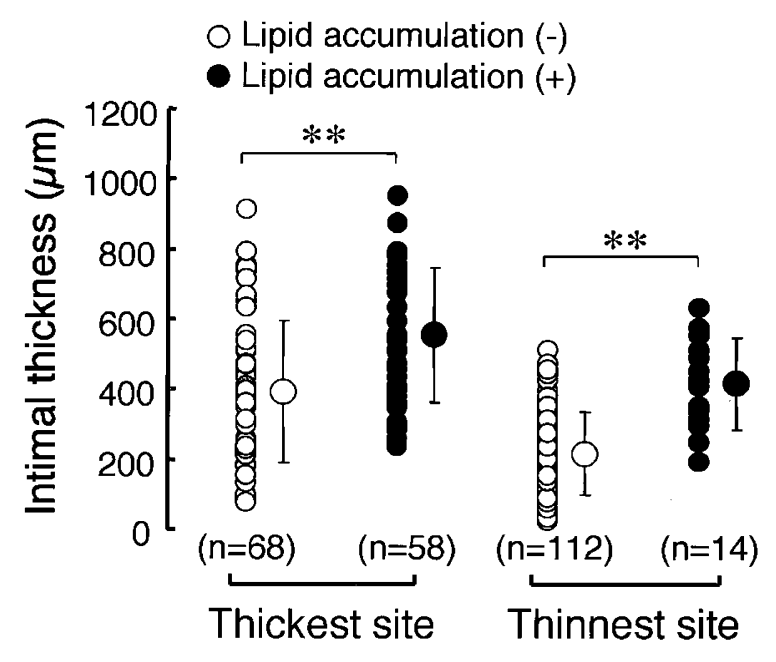

Figure 5.

Distribution of intimal thickness at the thickest and thinnest sites in each DIT section in terms of the presence or absence of lipid accumulation in the deep intimal layers. Coronary segments with lipid accumulation only in the subendothelium were excluded. Large symbols and vertical bars indicate means \pm SD. ${ }^{* \star} p<0.01$ by unpaired Student's $t$ test.

deep layers of DIT in which only a few or no macrophages (CD68-positive) were present (Fig. 3E). This deep intimal lipid accumulation was characterized by scattered fine lipid droplets compared with dense large ones within macrophages (Fig. 3, I versus J). In addition, advanced lesions often involved a similar lipid accumulation located deeply in the plaques, which was apparently distinct from the subendothelial lesions (Fig. 4C). These observations suggest that lipid accumulation localized deep in the thickened intima may occur independently of fatty streak formation. Our observations were obtained from middle- to advanced-aged people and may not be applicable to younger people in whom DIT is still immature (Ikari et al, 1999; Neufeld et al, 1962; Velican and Velican, 1979). Only small lipid deposits and macrophages in the subendothelium have been shown to be early intimal lesions in younger people (Napoli et al, 1997; Stary, 1989; Stary et al, 1992). It is also noted that lipid accumulation localized deep in DIT was found even in patients who had no coronary risk factors. This is consistent with a recent PDAY study (Ishikawa et al, 2001) in which the extent of apolipoprotein deposition in the human aortic intima has been shown to be increased with age but not correlated with the serum cholesterol levels. Although a regression of atherosclerosis resulting in lipid accumulation alone may have occurred under end-stage exhaustive conditions, the serum cholesterol levels in our cases were within the normal range until 1 month before death. The disappearance of macrophages in experimentally induced atherosclerosis has been shown to occur 6 to 12 months after the withdrawal of cholesterol (Kockx et al, 1998) or after treatment with pravastatin (Shiomi et al, 1995). It is possible that normal serum cholesterol levels in human (130-220 mg/dl) may be high enough to cause LDL deposition in the DIT because it is reported that the accumulation of LDL in the swine coronary artery DIT occurs in hyper- $(500 \mathrm{mg} / \mathrm{dl})$ but not normocholesterolemia (100 mg/dl) (Yamauchi and Hoff, 1984).

\section{8-iso-PGF ${ }_{2 \alpha}$ Formation in the Intimal LDL}

Several recent studies have shown that the amount of 8-iso-PGF $2 \alpha$ is increased in human atherosclerotic lesions including those of human coronary arteries (Gniwotta et al, 1997; Mehrabi et al, 1999; Praticò et al, 1997). The present study provides further evidence that immunoreactive 8 -iso-PGF $2 \alpha$ is extensively present even in human coronary DIT involving no apparent fatty streak formation. The 8-iso-PGF ${ }_{2 \alpha}$ staining was colocalized with apo B 100 staining in most lipid-rich areas, except that immunoreactive 8-iso- $\mathrm{PGF}_{2 \alpha}$ relative to apo B 100 was preferentially located in the deep layers of DIT (Fig. 2A). By oxidative pretreatment of sections with $20 \mu \mathrm{M} \mathrm{CuCl} \mathrm{Cl}_{2}$, we confirmed that the staining intensity for apo $\mathrm{B} 100$ and 8-iso- $\mathrm{PGF}_{2 \alpha}$ reciprocally reflected the local oxidized state, consistent with the results of in vitro studies using isolated LDL exposed to oxidative stress (Fong et al, 1987; Lynch et al, 1994; Morrow et al, 1992). These results suggest that oxidative modification of plasma-derived LDLs may occur slowly in the deep layers of DIT without cellular uptake or processing by macrophages. Also, in fibrofatty lesions, we observed strong staining for apo B 100 or 8 -iso-PGF $2 \alpha$ in the lipid-rich core. On the other hand, little staining was seen in areas with macrophage accumulation in spite of dense lipid droplets within those cells (Fig. 3J). Most LDL particles up-taken by macrophages would be oxidized and degraded, resulting in the loss of apo B 100 antigenicity (Brown and Goldstein, 1986; Steinberg and Lewis, 1997), and 8-iso-PGF ${ }_{2 \alpha}$ produced within cells could be rapidly liberated by the action of cellular phospholipase $A_{2}$ (Morrow et al, 1992). Our results are consistent with previous studies (Bocan and Guyton, 1985; Guyton and Klemp, 1994; Small et al, 1984; Smith et al, 1967) showing that lipid compositions differ between fatty streaks (intracellular) and the underlying lipid-rich core (extracellular) in some physicochemic properties.

\section{Possible Determinants for the Lipid Localization in DIT}

Major extracellular matrix components including collagen, elastin, and small proteoglycans biglycan and decorin have been established as LDL-retentive molecules (Kovanen and Pentikäinen, 1999; O'Brien et al, 1998; Podet et al, 1991; Williams and Tabas, 1995) and have been demonstrated in human coronary atherosclerotic plaques (O'Brien et al, 1998; Riessen et al, 1994). In the present study, we observed that type I and type III collagen, $\alpha$-elastin, and decorin were differentially expressed with the progression of lipid accumulation in the early stage of human coronary atherosclerosis (Fig. 4). This finding argues against the possibility that an extracellular matrix alteration in DIT may occur primarily before the lipid accumulation. Although hypoxic conditions may be present in the 

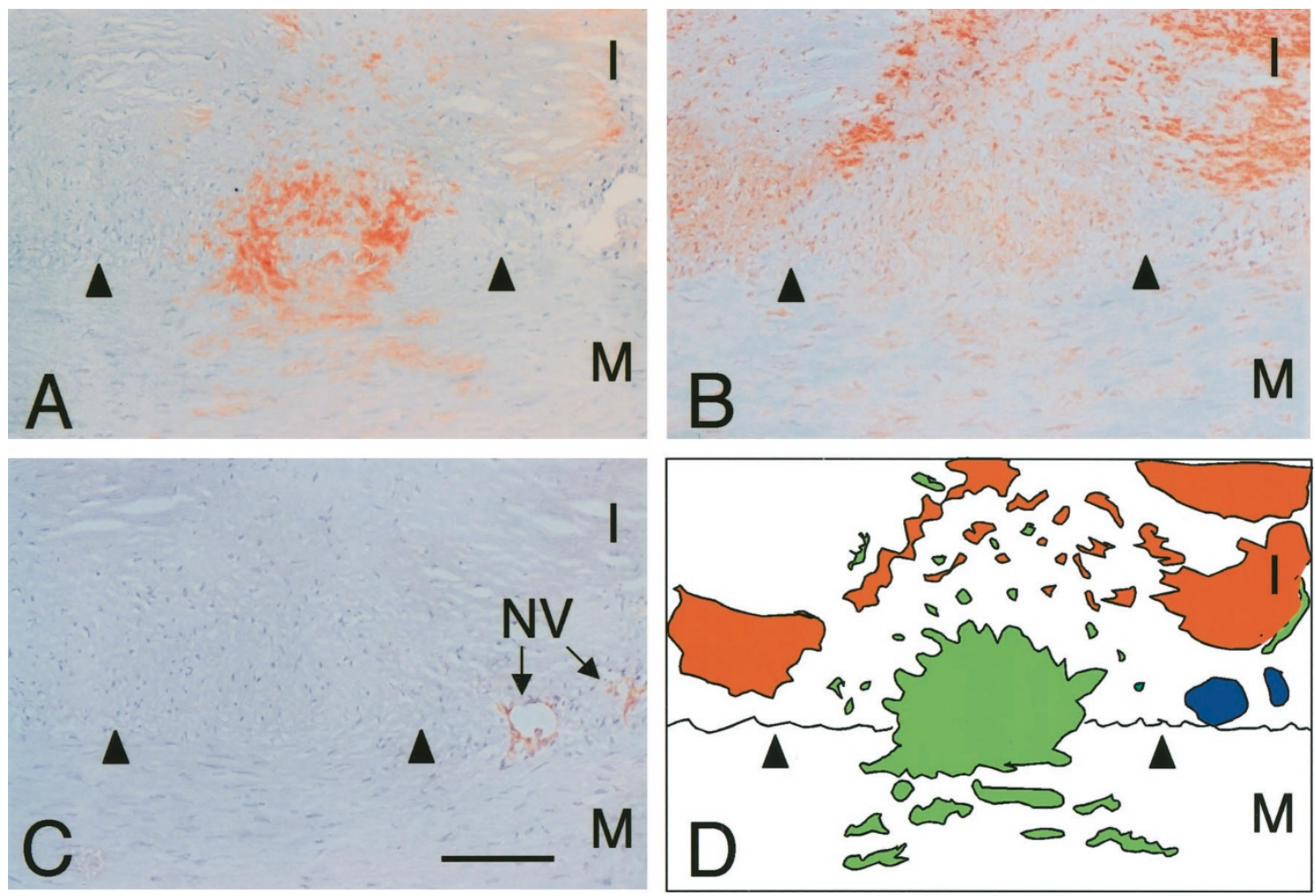

\section{Figure 6.}

Reciprocal immunohistochemic localization of apo B 100 and 8-iso- $\mathrm{PGF}_{2 \alpha}$ in the peripheral region of markedly thickened intima. A to $\mathrm{C}$ show serial sections stained for apo B 100 (A), 8-iso-PGF $2 \alpha$ (B), and CD31 (C). D shows a schema representing the relative location of apo B 100 (green), 8-iso-PGF $2 \alpha$ (red), and neovessels (blue). Arrowheads indicate internal elastic lamina. Bar $=100 \mu \mathrm{m} ; I=$ intima; $M=$ media; $N V=$ neovessel.

deep layers of DIT, it has been reported that hypoxia increases proteoglycan synthesis but decreases collagen synthesis in cultured smooth muscle cells (Pietilä and Jaakkola, 1984). This in vitro study does not support the possible effect of hypoxia itself on the extracellular matrix alteration seen in the deep intimal layers (Fig. 4B). Alternatively, oxidized LDL at lower concentrations has been shown to induce collagen synthesis in arterial smooth muscle cells involving neither proliferation nor apoptosis (Bachem et al, 1999; Jimi et al, 1995). This may be true in so far as oxidative modification of LDL progresses slowly in the deep layers of DIT. The observation that no apoptotic cells were found in the deep intimal layers containing oxidized lipid (Fig. 3K) also supports this possibility. Furthermore, it has been reported that LDL can stimulate glycosaminoglycan secretion by cultured fibroblasts and arterial smooth muscle cells depending on the proliferative state (Wosu et al, 1983). Thus, our observations are more compatible with the idea that increased synthesis of extracellular matrix molecules may result from LDL deposition and its oxidation in the intima. Nonetheless, once the extracellular matrix alterations have occurred in the intima, lipid accumulation would be accelerated.

Although an intimate relationship between lipid accumulation and nonatherosclerotic intimal thickening has been previously reported (Adams and Bayliss, 1969; Guyton et al, 1985; Ishikawa et al, 2001; Smith et al, 1967; Spring and Hoff, 1989; Yamauchi and Hoff,
1984), we report that lipid accumulation occurs preferentially in the deep layers rather than in the subendothelial layers of coronary intima in patients with normal serum cholesterol levels. In addition, intimal thickening greater than $200 \mu \mathrm{m}$ was required for the initiation of apparent lipid accumulation (Fig. 5). The physiologically thickened intima is nourished by diffusion from the luminal side and by the vasa vasorum from the adventitial side (Neufeld et al, 1962; Sims, 1989; Velican and Velican, 1979). As the intimal thickening progresses with age, some physical or metabolic abnormalities have been shown to occur in the nutritional border zone (Adams and Bayliss, 1969). In the present study, several lines of evidence support the idea that lipid accumulation occurs in the nutritional border zone of the thickened intima. First, lipid accumulation was inevitably localized in the deep intimal layers focally involving the inner media. Reciprocal staining from apo B 100-dominant to 8-isoPGF $_{2 \alpha}$-dominant toward the intima (Fig. 6D) suggests the perfusion from the adventitial side. Second, neovessel formation was occasionally found around the lipid accumulation. This would reflect that the areas involved with lipid accumulation are too remote to be nourished by direct diffusion from the luminal side. Given these observations, we speculate that an imbalance between the influx and efflux of the plasmaderived lipid may occur in the nutritional border zone (Nielsen, 1999). Although DIT greater than $200 \mu \mathrm{m}$ was not necessarily associated with lipid accumulation 


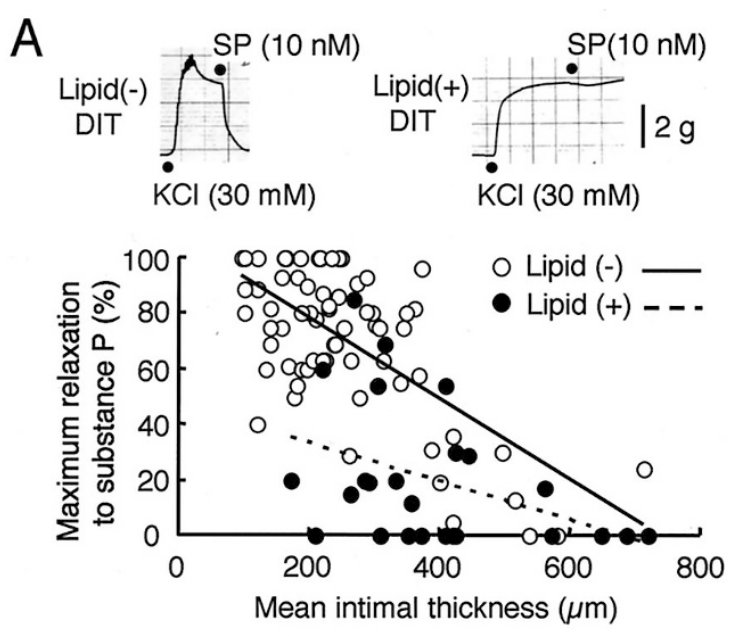

B

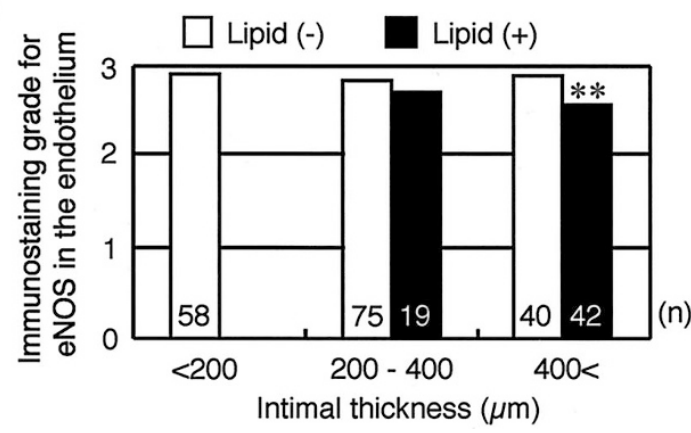

Figure 7.

Attenuated endothelium-dependent relaxation in human coronary arteries involved with deep intimal lipid accumulation. A shows typical responses to substance $\mathrm{P}(\mathrm{SP})$ in coronary rings with and without lipid accumulation and a plot of the relation between the extent of DIT and the maximum relaxation to substance $\mathrm{P}(10 \mathrm{~nm})$. Plots were significantly different between coronary rings with and without lipid accumulation ( $p<0.01$ by analysis of covariance). B shows the association of the extent of DIT and the presence of lipid accumulation with the endothelial nitric oxide synthase (eNOS) expression in the overlying endothelium. Each bar indicates mean score of cases belonging to each category. ${ }^{* \star} p<0.01$ versus comparable DIT cases by the MannWhitney $U$ test.

(Fig. 5), the cellular and extracellular environments in the deep intimal layer would be more critical than the extent of DIT itself.

\section{Role of the Deep Intimal Lipid Accumulation}

The presence of deep intimal lipid accumulation may in part explain the impaired endothelium-dependent relaxation that has been shown to be correlated with the extent of DIT in human coronary arteries (Keitoku et al, 1990). The critical intimal thickness at which the lipid accumulation appeared $(200 \mu \mathrm{m})$ was comparable with that at which attenuation of the endotheliumdependent relaxation to substance $\mathrm{P}$ occurred (Fig. $7 \mathrm{~A})$. At least in the DIT range from 200 to $400 \mu \mathrm{m}$, the expression of eNOS in the endothelium was preserved in most sections with and without lipid accumulation (Fig. 7B), consistent with the previous study (Fukuchi and Giaid, 1999). Oxidized LDL, mainly via lysophosphatidylcholine, has been shown to directly inhibit nitric oxide-stimulated cyclic guanosine monophos- phate accumulation in a bioassay system (Chin et al, 1992). Thus, it is possible that oxidized LDL present deep in DIT may act as an inactivating barrier to endothelium-derived relaxing factor before it reaches the media. However, when DIT was greater than 400 $\mu \mathrm{m}$, most coronary rings irrespective of the presence or absence of lipid accumulation showed only slight relaxation responses to substance $P$ (Fig. 7A). The deep intimal lipid accumulation was also found in fibrofatty or more advanced lesions with markedly thickened intima. This lipid accumulation combined with the subendothelial fatty lesions often showed dual lipid-rich layers across the intima (Fig. 4C). These observations suggest that different lipid accumulation processes that begin in the subendothelial or deep intimal regions may contribute to the complicated atheroma core formations in human coronary arteries.

\section{Conclusions}

In the epicardial human coronary arteries, we demonstrated for the first time substantial accumulation of normal and oxidized LDLs localized deep in the established DIT involving neither macrophage accumulation nor apoptosis in the constituent cells. This lipid accumulation in the DIT clearly coincided with increased type I and type III collagen and elastic fibers but rarely with sulfated proteoglycans including decorin. These results suggest that the accumulation of plasmaderived lipid and its oxidation in the DIT may occur in association with local extracellular matrix alterations but independently of inflammatory or apoptotic processes. We hypothesize that, although DIT occurs as a physiologic response early in life, its extraordinary development with age may induce some LDLretentive property in the nutritional border zone. Furthermore, we demonstrated that the presence of deep intimal lipid accumulation was associated with the reduced endothelium-dependent relaxation in human coronary arteries. The deep intimal lipid accumulation may contribute to the functional and morphologic abnormalities seen in human coronary atherogenesis that progresses slowly with age.

\section{Materials and Methods}

\section{Human Subjects and Preparation of Arteries}

This study was performed with the permission of the Ethics Committee of Tohoku University for clinical and experimental investigations. Epicardial human coronary arteries were obtained at autopsy from $17 \mathrm{pa}-$ tients ( 11 men and 6 women, 32-79 years old). The causes of death were cancer in nine patients, idiopathic cardiomyopathy in three patients, systemic lupus erythematosus in two patients, and amyotrophic lateral sclerosis, pulmonary fibrosis, and lung abscess in one patient each. No patients died of or had suffered from coronary artery disease, but 9 of 17 patients had one or more coronary risk factors including hypertension, diabetes mellitus, hyperlipidemia, and smoking. Coronary arteries were isolated 2 to 3 hours after death and were separated for histologic 
study and isometric tension recording. For histologic study, coronary segments were immediately fixed with $4 \%$ paraformaldehyde containing $0.1 \mathrm{~mm}$ butylated hydroxytoluene and $1 \mathrm{~mm}$ EDTA to minimize oxidative modifications of the arterial tissue. Serial cryostat sections ( $4 \mu \mathrm{m}$ thick) were cut every $3 \mathrm{~mm}$ along the arterial course. For isometric tension recording, coronary segments were placed in iced Krebs-Ringer solution containing the compositions as previously reported (Keitoku et al, 1990).

\section{Immunohistochemistry}

Immunohistochemistry was performed in cryostat sections by the ABC method as described previously (Fukuchi and Giaid, 1999). Briefly, sections were incubated serially with the following solutions: (a) $2 \%$ hydrogen peroxide for 30 minutes to block endogenous peroxidase activity, (b) $0.3 \%$ Triton-X 100 for 15 minutes, (c) $10 \%$ normal goat serum for 60 minutes, (d) primary antisera for 16 hours at $4^{\circ} \mathrm{C}$, (e) biotinylated goat anti-mouse or goat anti-rabbit $\lg G$ at a dilution of 1:200 for 45 minutes, and (f) avidinbiotinylated horseradish peroxidase complex (Vectastain; Vector Laboratories, Burlingame, California) at a dilution of 1:100 for 45 minutes. Immunoreactive sites were visualized by incubation with $0.025 \% 3$,3diaminobenzidine and $0.01 \%$ hydrogen peroxide for 3 minutes. PBS ( $\mathrm{pH} \mathrm{7.4)} \mathrm{was} \mathrm{used} \mathrm{to} \mathrm{dilute} \mathrm{each} \mathrm{solution}$ and to wash the sections three times between each step. Finally, tissue sections were counterstained with hematoxylin.

To identify plasma-derived normal and oxidized lipids, we used mouse anti-apo B 100 IgG (ICN Pharmaceuticals, Inc., Aurora, Ohio) and rabbit anti8-iso-PGF ${ }_{2 \alpha}$ polyclonal antibody (Cayman Chemical, Ann Arbor, Michigan), respectively. The specificity of 8 -iso-PGF ${ }_{2 \alpha}$ staining was assessed by preincubation of the antiserum with excess amounts of antigens and by pretreatment of the sections with $20 \mu \mathrm{M} \mathrm{CuCl}_{2}$ for 6 hours to expose the tissue-resident LDL to oxidative stress (Lynch et al, 1994). Lipid accumulation was also assessed by oil red $\mathrm{O}$ staining in serial sections. To identify the cell types, serial sections were immunostained for the endothelial cell marker CD31 (Dako, Glostrup, Denmark), smooth muscle actin (Enzo Diagnostics Inc., Farmingdale, New York), and macrophage CD68 (Dako). Extracellular matrix components were identified by using rabbit anti-type I collagen polyclonal antibody (Quartett, Berlin, Germany), mouse anti-type III collagen IgG (BioGenex, San Ramon, California), rabbit anti- $\alpha$-elastin polyclonal antibody (Biogenesis, Kingston, New Hampshire), and mouse anti-decorin IgG (R\&D Systems, Minneapolis, Minnesota). Sulfated proteoglycans were stained with Alcian blue $(\mathrm{pH} \mathrm{1.0)}$ in serial sections. Endothelial expression of eNOS was assessed by staining with mouse anti-eNOS IgG (Transduction Laboratories, Lexington, Kentucky). The specificity of immunostaining was confirmed by incubation with the nonimmune mouse or rabbit serum instead of the primary antiserum.

\section{Terminal Deoxynucleotidyltransferase-Mediated dUTP-Fluorescein Nick-End Labeling}

Apoptotic cells in situ were identified by using TUNEL as previously described (Gavrieli et al, 1992). Nuclei of tissue sections were stripped from proteins by incubation with $10 \mu \mathrm{g} / \mathrm{ml}$ proteinase $\mathrm{K}$ (Dako) for 30 minutes at room temperature. After washing in PBS, the slides were incubated with a reaction buffer containing terminal deoxynucleotidyltransferase and fluorescein-labeled dUTP (Boehringer Mannheim, Mannheim, Germany) at $37^{\circ} \mathrm{C}$ for 60 minutes. Negative control sections were incubated in the absence of the terminal deoxynucleotidyl transferase enzyme. After terminating the reaction in PBS, the sections were covered with $2 \%$ aqueous BSA for 30 minutes and then incubated with a solution of alkaline phosphatase-labeled anti-fluorescein Fab fragment (Boehringer Mannheim) at $37^{\circ} \mathrm{C}$ for 30 minutes. After washing in PBS for 15 minutes, the sections were visualized with Fuchsin substrate-chromogen system (Dako) and were counterstained with hematoxylin. Positive staining was confirmed in control slides pretreated with DNase I (32 U/ml acetate buffer; Sigma, St. Louis, Missouri) for 10 minutes before starting the TUNEL method.

\section{Isometric Tension Recording}

Isometric tension recording was performed as previously described (Keitoku et al, 1990). Briefly, a coronary ring segment of $2.5 \mathrm{~mm}$ in width was suspended in a tissue bath containing $10 \mathrm{ml}$ Krebs-Ringer solution and connected to a tension transducer (Shinkoh U-Gauge, type UL-20; Shinkoh, Tokyo, Japan). The bath solution was saturated with $95 \% \mathrm{O}_{2} / 5 \% \mathrm{CO}_{2}$ at $37^{\circ} \mathrm{C}$ and the $\mathrm{pH}$ was 7.40 . Isometric tension was recorded on an oscillograph (Rectigraph-8S; San-ei, Tokyo, Japan). During the 3 hours allowed for equilibration, the resting tension was adjusted to $1.5 \mathrm{~g}$, the optimal tension for inducing the maximal contraction with $50 \mathrm{~mm} \mathrm{KCl}$. Maximum endothelium-dependent relaxation was assessed by adding $10 \mathrm{~nm}$ substance $P$ to each ring precontracted with $30 \mathrm{mM} \mathrm{KCl}$ that produced a tension approximating $30 \%$ of the maximum $\mathrm{KCl}$-induced contractions. The relaxation response was expressed as a percentage of $100 \mu \mathrm{M}$ papaverine-induced relaxation. After the pharmacologic experiments, the ring segments were prepared for histologic examination to evaluate the lipid accumulation and the extent of DIT.

\section{Histologic Analysis}

Each coronary segment was classified into four atherosclerotic types: DIT, fibrofatty lesion, fibrous plaque, and advanced plaque, as defined by Gown et al (1986). In the present study, DIT was characterized by the preserved tissue structure and smooth muscle cellularity and by only a few scattered or no macrophages positive to CD68 throughout the intima. In DIT sections stained with hematoxylin and eosin, the intimal thickness $(\mu \mathrm{m})$ was measured as the distance from the luminal surface to the internal elastic lamina 
at the thickest and thinnest sites around the circumference. Mean intimal thickness was calculated as the mean of the thickest and thinnest sites of DIT in each coronary segment. Immunostaining intensity for eNOS in the endothelium was semiquantitatively graded from 0 to 3 in each atherosclerotic lesion $(0$ for absence of staining, 1 for only focal or weak, 2 for diffusely moderate, and 3 for diffusely strong). In the DIT section, eNOS staining was graded individually around the thickest and thinnest sites. The grading was performed independently by two observers and was finally determined by joint observation. The TUNEL-positive cells per section were counted independently of classifying the atherosclerotic types and localizing the lipid accumulation in the intima.

\section{Statistical Analysis}

The incidence of DIT among all coronary segments, the prevalence of lipid accumulation in DIT, and alterations in extracellular matrix molecules associated with lipid accumulation were compared among different groups by chi-square analysis. The intimal thickness between coronary segments with and without lipid accumulation in the DIT was compared by unpaired Student's $t$ test. A plot of the relation between the intimal thickness and the maximum relaxation response to substance $\mathrm{P}$ was compared between two groups by analysis of covariance. Immunohistochemic grades for eNOS were compared among different groups by the Kruskal-Wallis rank test or between two groups by the Mann-Whitney $U$ test. Values of $p<0.05$ were considered significant.

\section{Acknowledgments}

We thank Mr. Brent Bell for reading the manuscript. Autopsy heart tissues were supplied by the Department of Pathology of Tohoku University Graduate School of Medicine.

\section{References}

Adams CW and Bayliss OB (1969). The relationship between diffuse intimal thickening, medial enzyme failure and intimal lipid deposition in various human arteries. $\mathrm{J}$ Atheroscler Res 10:327-339.

Bachem MG, Wendelin D, Schneiderhan W, Haug C, Zorn U, Gross HJ, Schmid-Kotsas A, and Grünert A (1999). Depending on their concentration oxidized low density lipoproteins stimulate extracellular matrix synthesis or induce apoptosis in human coronary artery smooth muscle cells. Clin Chem Lab Med 37:319-326.

Bocan TM and Guyton JR (1985). Human aortic fibrolipid lesions: Progenitor lesions for fibrous plaques, exhibiting early formation of the cholesterol-rich core. Am J Pathol 120:193-206.

Brown MS and Goldstein JL (1986). A receptor-mediated pathway for cholesterol homeostasis. Science 232:34-47.

Chin JH, Azhar S, and Hoffman BB (1992). Inactivation of endothelial derived relaxing factor by oxidized lipoproteins. $\mathrm{J}$ Clin Invest 89:10-18.
Eggen DA and Solberg LA (1968). Variation of atherosclerosis with age. Lab Invest 18:571-579.

Fong LG, Parthasarathy S, Witztum JL, and Steinberg D (1987). Nonenzymatic oxidative cleavage of peptide bonds in apoprotein B-100. J Lipid Res 28:1466-1477.

Fukuchi M and Giaid A (1999). Endothelial expression of endothelial nitric oxide synthase and endothelin-1 in human coronary artery disease. Lab Invest 79:659-670.

Gavrieli Y, Sherman Y, and Ben-Sasson SA (1992). Identification of programmed cell death in situ via specific labeling of nuclear DNA fragmentation. J Cell Biol 119:493-501.

Gniwotta C, Morrow JD, Roberts LJ, and Kühn H (1997). Prostaglandin $F_{2}$-like compounds, $F_{2}$ isoprostanes, are present in increased amounts in human atherosclerotic lesions. Arterioscler Thromb Vasc Biol 17:3236-3241.

Gordon D, Reidy MA, Benditt EP, and Schwartz SM (1990). Cell proliferation in human coronary arteries. Proc Natl Acad Sci USA 87:4600-4604.

Gown AM, Tsukada T, and Ross R (1986) Human atherosclerosis II immunocytochemical analysis of the cellular composition of human atherosclerotic lesions. Am J Pathol 125: 191-207.

Guyton JR, Bocan TMA, and Schifani TA (1985) Quantitative ultrastructural analysis of perifibrous lipid and its association with elastin in nonatherosclerotic human aorta. Arteriosclerosis $5: 644-652$.

Guyton JR and Klemp KF (1994). Development of the atherosclerotic core region: Chemical and ultrastructural analysis of microdissected atherosclerotic lesions from human aorta. Arterioscler Thromb 14:1305-1314.

Ikari Y, McManus BM, Kenyon J, and Schwartz SM (1999). Neonatal intima formation in the human coronary artery. Arterioscler Thromb Vasc Biol 19:2036-2040.

Ishikawa $\mathrm{Y}$, Ishii T, Akasaka $\mathrm{Y}$, Masuda T, Strong JP, Zieske AW, Takei H, Malcom GT, Taniyama M, Choi-Miura $\mathrm{NH}$, and Tomita M (2001). Immunolocalization of apolipoproteins in aortic atherosclerosis in American youths and young adults: Findings from the PDAY study. Atherosclerosis 158:215-225.

Jimi S, Saku K, Uesugi N, Sakata N, and Takebayashi S (1995). Oxidized low density lipoprotein stimulates collagen production in cultured arterial smooth muscle cells. Atherosclerosis 116:15-26.

Keitoku M, Okayama H, Satoh $\mathrm{Y}$, Maruyama $\mathrm{Y}$, and Takishima $T$ (1990). Supersensitivity to histamine in early atherosclerotic human coronary arteries: Intimal thickening attenuates endothelium-dependent relaxation. J Appl Cardiol 5:101-114.

Kockx MM, De Meyer GR, Buyssens N, Knaapen MW, Bult H, and Herman AG (1998). Cell composition, replication, and apoptosis in atherosclerotic plaques after 6 months of cholesterol withdrawal. Circ Res 83:378-387.

Kovanen PT and Pentikäinen MO (1999). Decorin links lowdensity lipoproteins (LDL) to collagen: A novel mechanism for retention of $L D L$ in the atherosclerotic plaque. Trends Cardiovasc Med 9:86-91.

Lynch SM, Morrow JD, Roberts LJ, and Frei B (1994). Formation of non-cyclooxygenase-derived prostanoids $\left(F_{2^{-}}\right.$ isoprostanes) in plasma and low density lipoprotein exposed to oxidative stress in vitro. J Clin Invest 93:998-1004. 
Mehrabi MR, Ekmekcioglu C, Tatzber F, Oguogho A, Ullrich R, Morgan A, Tamaddon F, Grimm M, Glogar HD, and Sinzinger $\mathrm{H}$ (1999). The isoprostane, 8-epi-PGF ${ }_{2 \alpha}$, is accumulated in coronary arteries isolated from patients with coronary heart disease. Cardiovasc Res 43:492-499.

Montenegro MR and Eggen DA (1968). Topography of atherosclerosis in the coronary arteries. Lab Invest 18:586-593.

Morrow JD, Awad JA, Boss HJ, Blair IA, and Roberts LJ (1992). Non-cyclooxygenase-derived prostanoids $\left(F_{2^{-}}\right.$ isoprostanes) are formed in situ on phospholipids. Proc Natl Acad Sci USA 89:10721-10725.

Napoli C, D'Armiento FP, Mancini FP, Postiglione A, Witztum JL, Palumbo G, and Palinski W (1997). Fatty streak formation occurs in human fetal aortas and is greatly enhanced by maternal hypercholesterolemia. J Clin Invest 100:26802690.

Neufeld HN, Wagenvoort CA, and Edwards JE (1962). Coronary arteries in fetuses, infants, juveniles, and young adults. Lab Invest 11:837-844.

Nielsen LB (1999). Atherogenicity of lipoprotein (a) and oxidized low density lipoprotein: Insight from in vivo studies of arterial wall influx, degradation and efflux. Atherosclerosis 143:229-243.

O'Brien KD, Olin KL, Alpers CE, Chiu W, Ferguson M, Hudkins K, Wight TN, and Chait A (1998). Comparison of apolipoprotein and proteoglycan deposits in human coronary atherosclerotic plaques. Circulation 98:519-527.

Pathobiological Determinants of Atherosclerosis in Youth (PDAY) Research Group (1993). Natural history of aortic and coronary atherosclerotic lesions in youth: Findings from the PDAY study. Arterioscler Thromb 13:1291-1298.

Pietilä K and Jaakkola O (1984). Effect of hypoxia on the synthesis of glycosaminoglycans and collagen by rabbit aortic smooth muscle cells in culture. Atherosclerosis 50: 183-190.

Podet EJ, Shaffer DR, Gianturco SH, Bradley WA, Yang CY, and Guyton JR (1991). Interaction of low density lipoproteins with human aortic elastin. Arterioscler Thromb 11:116-122.

Praticò D, luliano L, Mauriello A, Spagnoli L, Lawson JA, Maclouf J, Violi F, and FitzGerald GA (1997). Localization of distinct $F_{2}$-isoprostanes in human atherosclerotic lesions. J Clin Invest 100:2028-2034.

Riessen R, Isner JM, Blessing E, Loushin C, Nikol S, and Wight TN (1994). Regional differences in the distribution of the proteoglycans biglycan and decorin in the extracellular matrix of atherosclerotic and restenotic human coronary arteries. Am J Pathol 144:962-974.

Ross R (1999). Atherosclerosis: An inflammatory disease. N Engl J Med 340:115-126.
Schwartz SM, deBlois D, and O'Brien ER (1995). The intima: Soil for atherosclerosis and restenosis. Circ Res 77:445-465.

Shiomi M, Ito T, Tsukada T, Yata T, Watanabe Y, Tsujita Y, Fukami M, Fukushige J, Hosokawa T, and Tamura A (1995). Reduction of serum cholesterol levels alters lesional composition of atherosclerotic plaques: Effect of pravastatin sodium on atherosclerosis in mature WHHL rabbits. Arterioscler Thromb Vasc Biol 15:1938-1944.

Sims FH (1989). A comparison of structural features of the walls of coronary arteries from 10 different species. Pathology 21:115-124.

Small DM, Bond MG, Waugh D, Prack M, and Sawyer JK (1984). Physicochemical and histological changes in the arterial wall of nonhuman primates during progression and regression of atherosclerosis. J Clin Invest 73:1590-1605.

Smith EB, Evans PH, and Downham MD (1967). Lipid in the aortic intima: The correlation of morphological and chemical characteristics. J Atheroscler Res 7:171-186.

Spring PM and Hoff HF (1989). LDL accumulation in the grossly normal human iliac bifurcation and common iliac arteries. Exp Mol Pathol 51:179-185.

Stary HC (1989). Evolution and progression of atherosclerotic lesions in coronary arteries of children and young adults. Arteriosclerosis (Suppl 19):I19-I32.

Stary HC, Blankenhorn DH, Chandler AB, Glagov S, Insull W Jr, Richardson M, Rosenfeld ME, Schaffer SA, Schwartz CJ, Wagner WD, and Wissler RW (1992). A definition of the intima of human arteries and of its atherosclerosis-prone regions: $\mathrm{A}$ report from the Committee on Vascular Lesions of the Council on Arteriosclerosis, American Heart Association. Arterioscler Thromb 12:120-134.

Steinberg D and Lewis A (1997). Oxidative modification of LDL and atherogenesis. Circulation 95:1062-1071.

Velican C and Velican D (1979). Some particular aspects of the microarchitecture of human coronary arteries. Atherosclerosis 33:191-200.

Williams KJ and Tabas I (1995) The response-to-retention hypothesis of early atherogenesis. Arterioscler Thromb Vasc Biol 15:551-561.

Wosu L, Parisella R, and Kalant N (1983). Effect of low density lipoprotein on glycosaminoglycan secretion by cultured human smooth muscle cells and fibroblasts: Influence of serum concentration and cell proliferation rate. Atherosclerosis $48: 205-220$.

Yamauchi $Y$ and Hoff HF (1984). Apolipoprotein B accumulation and development of foam cell lesions in coronary arteries of hypercholesterolemic swine. Lab Invest 51:325332. 